\title{
Probabilistic Sensitivities for Fatigue Analysis of Turbine Engine Disks
}

\author{
Harry R. Millwater and R. Wesley Osborn \\ Department of Mechanical Engineering, University of Texas at San Antonio, San Antonio, TX 78249, USA
}

Received 22 January 2006; Revised 21 June 2006; Accepted 3 August 2006

\begin{abstract}
A methodology is developed and applied that determines the sensitivities of the probability-of-fracture of a gas turbine disk fatigue analysis with respect to the parameters of the probability distributions describing the random variables. The disk material is subject to initial anomalies, in either low- or high-frequency quantities, such that commonly used materials (titanium, nickel, powder nickel) and common damage mechanisms (inherent defects or surface damage) can be considered. The derivation is developed for Monte Carlo sampling such that the existing failure samples are used and the sensitivities are obtained with minimal additional computational time. Variance estimates and confidence bounds of the sensitivity estimates are developed. The methodology is demonstrated and verified using a multizone probabilistic fatigue analysis of a gas turbine compressor disk analysis considering stress scatter, crack growth propagation scatter, and initial crack size as random variables.
\end{abstract}

Copyright (c) 2006 H. R. Millwater and R. W. Osborn. This is an open access article distributed under the Creative Commons Attribution License, which permits unrestricted use, distribution, and reproduction in any medium, provided the original work is properly cited.

\section{INTRODUCTION}

As a result of the accident at Sioux City, Iowa, in 1989, the FAA requested in 1991 that industry review available techniques determine whether a damage tolerance approach could be introduced to reduce the rate of uncontained rotor events. The industry working group concluded that additional enhancements to the conventional rotor life management methodology could be established which explicitly address anomalous conditions. Henceforth, the FAA, industry, and research partners have been proactively developing the methodology [1-3], computer software [4, 5], and necessary data to quantify and hence mitigate the probabilityof-fracture (POF) of gas turbine engine rotor disks.

Significant research effort has been undertaken in order to determine needed input data for probabilistic fatigue analyses of gas turbine disks to be useful. As examples, though not exhaustive, the following input data have been developed: the frequency and probability distribution for the initial defect size of hard alpha particles in titanium [6, 7], the constitutive properties of hard alpha defects [8], the crack growth rate in a vacuum for titanium [8], fatigue testing of the initiation of cracking for hard alpha defects [9], effects of residual stresses around hard alpha particles [10], the expected amount of variation in fatigue crack propagation scatter [11], and weight-function-based stress intensity factor equations for flaws under a bivariant stress field [12]. An FAA advisory circular has been issued that describes a probabilistic methodology and suggested input data to quantify the POF with respect to hard alpha defects [13].

The methodology has been extended to surface damage, that is, damage induced during manufacturing, by addressing stress gradient modeling issues prevalent at the part surface and developing the requisite weight function stress intensity factor solutions [14]. The methodology and software have also been extended to materials with potentially highfrequency inherent anomalies such as in powder nickel by considering the potential for multiple anomalies in a disk. The frequency of anomalies is determined using a Poisson distribution with the mean occurrence rate determined from inspections of material [2].

Chan et al. [15] have developed microstructurally based probabilistic models that predict fracture parameters such as crack initiation life, crack growth life, fatigue crack growth threshold, and fracture toughness, among others, and have applied the methodology to Ti-6Al-4V. Material-specific random variables are considered such as grain size, slip band width, fatigue limit, and Taylor factor. At a higher modeling scale, probabilistic models for the Paris constant and exponent for Ti-6Al-4V have been developed and compared against experimental data [16]. In contrast, the methodology presented here is a higher level engineering-based approach 
in that the target user is a knowledgeable engineer or designer evaluating the POF of a proposed design. As such, the random variables considered are fewer and more inclusive.

Component lifetime is often segregated into "initiation" and "propagation" stages. The initiation life is sometimes further subdivided. For example, pit nucleation and pit growth are often explicitly addressed in corrosion instigated fatigue [17]. Hard alpha defects in titanium, however, are typically brittle and often have microcracks and microvoids, especially larger defects, and, therefore, crack growth with respect to hard alpha anomalies is assumed to begin at the first cycle of service loading [18], however, the probabilistic and fracture mechanics and sensitivity methodologies are being extended to include time-to-crack initiation in order to support analyses of other forms of damage in addition to hard alpha.

Probabilistically based sensitivity methods in probabilistic analysis have a long and storied history, particularly with respect to first/second-order reliability methods (FORM/ SORM). Sensitivity factors (derivatives of the safety index with respect to the random variables) [19], derivatives of the probability-of-fracture with respect to the random variable parameters, for example, $\partial P / \partial \mu, \partial P / \partial \sigma$ [19], and omission factors [20] are computed as byproducts of an analysis.

Variance-based methods $[21,22]$ have been applied to design under uncertainty problems [23, 24]. Variance-based methods are capable of identifying the contributions of the "main" and "interaction" effects and the total sensitivity index (the sum of all the sensitivity indices, including all the interaction effects) for any parameter upon the model output. Groups of variables can also be considered. The method requires multiple multidimensional integrals. The Fourier amplitude sensitivity test (FAST) method can be used to reduce the multidimensional integrals into one-dimensional integrals [23].

Sampling-based sensitivities, however, have received less attention. Karamchandani [25] presented the fundamental concept by directly differentiating the probability-of-failure integral with respect to the parameters of the random variables, for example, mean and standard deviation, then reformulating the modified integral as an expected value operator such that sampling methods can be used to compute the sensitivities. However, only the normal probability distribution was considered. Wu [26] generalized the concept to other probability distributions and extended it to system reliability problems and used importance sampling for the probability calculations. He also suggested a nondimensionalizing approach in order to compare probabilistic sensitivities across random variables.

In this research, we continue in a similar fashion to Karamchandani [25] and $\mathrm{Wu}$ [26], that is, we consider samplingbased (Monte Carlo) sensitivities of the probability-offracture estimates with respect to the parameters of the random variables, but address several new requirements such as (a) an initial crack size distribution that is of a tabular format and develops sensitivities with respect to the bounds of the distribution, (b) a zone-based methodology that accounts for distributed damage, and (c) variance estimates and hence confidence bounds for the probabilistic sensitivity estimates. A significant feature of the computation is that samplingbased sensitivities can be computed without any additional limit state calculations, and thus are very inexpensive to compute.

The paper is organized as follows. In the following section we outline briefly the essential mathematics for computing the probability-of-fracture of a gas turbine disk subject to (potentially) a rate of high-frequency anomalies. In the subsequent section, we derive the probabilistic sensitivities in a hierarchical fashion; with respect to the (a) disk, (b) unconditional POF of a zone, and (c) conditional (assumes a defect is present) POF of a zone. Variance estimates for the probabilistic sensitivities are next derived, again in a hierarchical fashion. Finally, a numerical example is presented of a hypothetical titanium compressor disk and the sensitivities are verified against finite difference estimates.

\section{PROBABILISTIC FATIGUE METHODOLOGY}

The probabilistic methodology employs a "zone-based" system reliability approach to compute the probability-offracture of a turbine disk as a function of flight cycles. For "inherent" defects, that is, defects contained in the material before manufacturing, for example, hard alpha, a zone represents a volume of material in which each subregion of a zone generally has a uniform stress state, the same properties, and anomaly distribution. In other words, the risk computed for each subregion of material in the zone will be the same; or nearly so, thus, the material is grouped into a zone and the probability-of-fracture for a defect originating anywhere in the zone is computed. In practice, a single "life limiting" location is chosen to locate all defects originating in a zone. For "induced" defects, that is, defects introduced during manufacturing or maintenance, a zone represents a "feature" such as a bolt hole surface. Again, a life limiting location of the feature is chosen as the location at which to locate all initial defects.

A concise summary of the governing mathematics is given below to serve as an outline for the subsequent differentiation of the equations in order to compute the sensitivities. More details regarding the methodology can be found in references $[2,3]$.

\subsection{Probabilistic treatment of multiple anomalies}

The probability-of-fracture within an individual zone, $P_{F, z o n e}$, can be computed given the knowledge of the mean anomaly occurrence rate $(\lambda)$ and the conditional POF $\left(P_{F C \text {,zone }}{ }^{-}\right.$ assumes a defect is present), as

$$
P_{F, \text { zone }}=1-\exp \left[-\lambda \cdot P_{F C \text {,zone }}\right] .
$$

$P_{F C \text {,zone }}$ is computed using Monte Carlo sampling and is hereafter denoted $P_{\mathrm{MC}}$ and is discussed later. $\lambda$ and $P_{\mathrm{MC}}$ are specific to each zone. 


\subsection{Disk risk}

The probability-of-fracture of the disk can be modeled as a series system of the zones:

$$
P_{F}=1-\prod_{k=1}^{m}\left[1-P_{F, k}\right]
$$

where $m$ equals the number of zones, and $P_{F, k}$ is given by (1).

\subsection{Random variables considered}

Three random variables are considered in the analysis: crack propagation scatter (uncertainty in the fracture mechanics calculations and material crack growth rate variability), stress scatter (uncertainty in the magnitude of the applied stresses), and the initial crack size.

\subsubsection{Propagation scatter}

Propagation scatter is a collection of uncertainties related to prediction of fatigue life such as the scatter in fatigue crack growth rate or the uncertainty in the numerical prediction of cycles-to-failure. As such, propagation scatter is modeled as a random variable $(B)$ that multiples the numerically predicted cycles-to-fracture,

$$
N_{f}=B \cdot N_{f}^{\prime},
$$

where $N_{f}$ is the cycles-to-failure used to estimate the probability-of-fracture, $N_{f}^{\prime}$ is the numerically predicted cycles-to-failure from a fracture mechanics algorithm, and $B$ is modeled using a two-parameter lognormal distribution described by a median and coefficient of variation (cov). Therefore, the sensitivity of the probability-of-fracture with respect to the median and cov of propagation scatter is computed.

Propagation scatter is modeled as an independent random variable for each zone since the error in fracture mechanics modeling may be feature, and, therefore, location independent. As a result, sensitivities with respect to median and cov are developed for each zone. If the median and cov values used are the same for each zone, a "cumulative" effect of the sensitivities can be developed that determines the sensitivity of the disk POF with respect to a single propagation scatter median and cov.

\subsubsection{Stress scatter}

Variations in applied stresses are simulated using a multiplier that can be considered random, that is,

$$
\sigma=S \cdot \sigma^{\prime}
$$

where $\sigma$ represents the stresses used in the calculation of the probability-of-fracture, $\sigma^{\prime}$ are the stresses input, for example, from a finite element analysis, and $S$ is modeled using a two-parameter lognormal distribution described by a median and coefficient of variation. Therefore, the sensitivity of

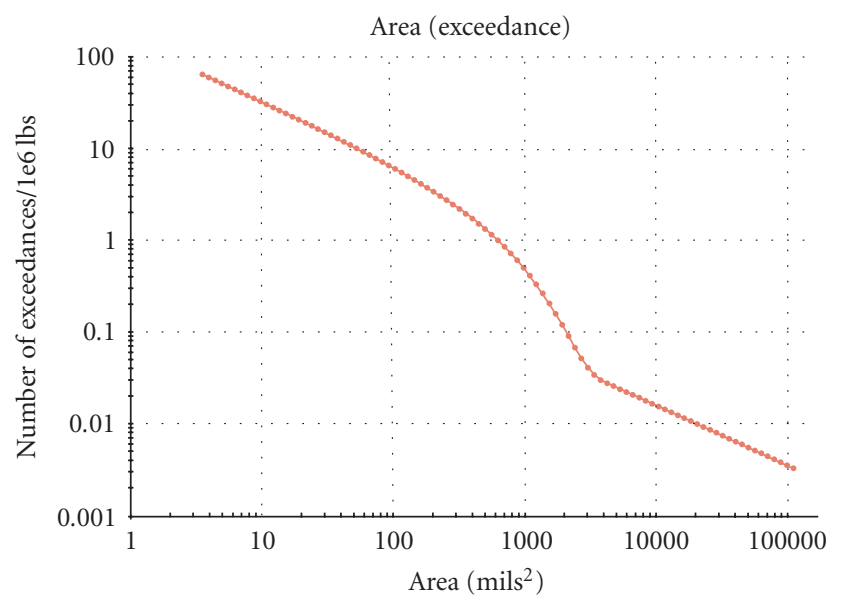

FIGURE 1: Exceedance curve: number of occurrence of anomalies versus crack size area per million pounds of titanium.

the probability-of-fracture with respect to the median and cov of stress scatter is computed.

Stress scatter is modeled as a fully dependent random variable across zones to simulate variability in inertia-based loading that affects all zones, for example, RPM. Therefore, a single sensitivity of the POF of the disk with respect to the median and cov of stress scatter is determined.

\subsubsection{Initial crack size: anomaly distribution}

The initial crack size is a critical element of predicting the probability-of-fracture. An anomaly distribution for hard alpha particles in titanium has been developed and calibrated by industry [6].

The format for the anomaly distribution is a tabular exceedance curve that presents the cumulative number of defects equal to or greater than a specific size, referenced to a weight, volume, or area of material. An example of an exceedance curve is shown in Figure 1. The cumulative distribution function, $F_{A}(a)$, and probability density function, $f_{A}(a)$, are

$$
F_{A}(a)=\frac{N\left(a_{\min }\right)-N(a)}{N\left(a_{\min }\right)-N\left(a_{\max }\right)},
$$

where $a_{\min }$ is the minimum crack size specified, $a_{\max }$ is the maximum crack size specified, $N[a]$ is the number of defects greater than or equal to size $a, N\left[a_{\max }\right]$ is the number of defects associated with the largest defect size, and $N\left[a_{\min }\right]$ is the total number of defects greater than or equal to the minimum crack size. The corresponding probability density function is given as

$$
f_{A}(a)=\frac{N\left(a_{i}\right)-N\left(a_{a_{i}+1}\right)}{a_{i}-a_{i+1}} \cdot \frac{1}{N\left(a_{\min }\right)-N\left(a_{\max }\right)},
$$

where $a_{i}$ and $a_{i+1}$ are the tabular values of defect size that bound crack size $a$. The units for describing the initial defect size can be given in terms of defect area, length, or depth. 
The exceedance curve also defines the probability of occurrence of a defect in that it is a function of a reference amount of material; either weight, volume, or area. For example, if the reference volume used to develop the exceedance curve is denoted $\Psi$, then the mean occurrence rate of a defect of any size in a zone of volume $\Psi_{i}$ is $\lambda_{i}=N\left(a_{\min }\right)$. $\Psi_{i} / \Psi$.

Critical components of modeling an exceedance curve are the specification of the bounds $a_{\min }$ and $a_{\max }$. These bounds define the range of crack sizes that will be considered in the analysis. As a result, probabilistic sensitivities with respect to the bounds of the exceedance curve, $a_{\min }$ and $a_{\max }$, are computed.

\section{PROBABILISTIC SENSITIVITIES FOR RANDOM VARIABLE STATISTICAL PARAMETERS}

Probabilistic sensitivities are defined as the partial derivative of the probability-of-fracture with respect to the parameters of the random variables. In the proceeding sections, the mathematics for computing probabilistic sensitivities will be given initially in general terms using the parameter $\theta$, where $\theta$ represents a parameter of a random variable, and then described in detail for the specific random variable parameters considered in this research.

\subsection{Sensitivity of the probability-of-fracture of the disk with respect to $\theta$}

The probability-of-fracture of the disk is given by (2), therefore, we compute the partial derivative of this equation with respect to the parameter $\theta$. Using the fact that $\prod_{k=1}^{m}(1-$ $\left.P_{F, k}\right)=1-P_{F}$, we obtain

$$
\frac{\partial P_{F}}{\partial \theta}=\left(1-P_{F}\right) \cdot \sum_{i=1}^{\hat{m}}\left(\frac{\partial P_{F, i}}{\partial \theta} \cdot \frac{1}{\left(1-P_{F, i}\right)}\right),
$$

where $\hat{m}$ is defined as the number of zones affected by the random variable which pertains to $\theta$. For stress scatter, $\hat{m}=$ $m$, for propagation scatter, $\hat{m}=1$, and for the exceedance curve, $\hat{m}$ equals number of zones that use a particular exceedance curve. Typically, only one exceedance curve is used per disk, and, in that case, $\hat{m}=m$ for the exceedance curve.

\subsection{Sensitivity of the probability-of-fracture per zone with respect to $\theta$}

From (7), the term $\partial P_{F, i} / \partial \theta$ is needed to compute the sensitivity of the disk; therefore, we compute the partial derivative of (1) with respect to $\theta$ as

$$
\frac{\partial P_{F, i}}{\partial \theta}=\left(1-P_{F, i}\right) \cdot\left(\frac{\partial \lambda_{i}}{\partial \theta} \cdot P_{\mathrm{MC}_{i}}+\lambda_{i} \cdot \frac{\partial P_{\mathrm{MC}_{i}}}{\partial \theta}\right),
$$

where $i$ denotes the zone number.

The term $\partial \lambda_{i} / \partial \theta$ is only nonzero when formulating the sensitivity with respect to $a_{\min }$ of the exceedance curve and is zero for all other sensitivities. This term defines the change in the probability of having a defect as a function of $a_{\min }$ and is negative; that is, increasing $a_{\min }$ will reduce the probability of having a defect. The resulting partial derivative is

$$
\begin{aligned}
\frac{\partial\left(\lambda_{i}\right)}{\partial a_{\min }} & =\frac{\partial N\left(a_{\min }\right)}{\partial a_{\min }} \cdot \frac{\Psi_{i}}{\Psi}=-f_{A}\left(a_{\min }\right) \cdot\left[N\left(a_{\min }\right)-N\left(a_{\max }\right)\right] \cdot \frac{\Psi_{i}}{\Psi} \\
& =-f_{A}\left(a_{\min }\right) \cdot\left[N\left(a_{\min }\right)-N\left(a_{\max }\right)\right] \cdot \frac{\lambda_{i}}{N\left(a_{\min }\right)} .
\end{aligned}
$$

\subsection{Sensitivity of the conditional probability- of-fracture per zone with respect to $\theta$}

$P_{\mathrm{MC}}$ is the conditional probability-of-fracture computed using Monte Carlo sampling,

$$
P_{\mathrm{MC}}=\int_{g(\mathbf{x}) \leq 0} f_{x}(\mathbf{x}) \cdot d \mathbf{x}
$$

where $\mathbf{x}$ is a vector of random variables, $f_{\mathbf{x}}(\mathbf{x})$ is the joint density function of $\mathbf{x}$, and $g$ is the limit state function defined such that $g(\mathbf{x}) \leq 0$ defines fracture.

Inserting the indicator function $I(\mathbf{x})$, defined as equal to one if $g(\mathbf{x}) \leq 0$ and zero otherwise,

$$
P_{\mathrm{MC}}=\int_{-\infty}^{\infty} I(\mathbf{x}) f_{\mathbf{x}}(\mathbf{x}) d \mathbf{x}
$$

We take the derivative of this equation in order to compute the partial derivative of $P_{\mathrm{MC}}$ with respect to a parameter of the random variable $j$, denoted $\theta_{j}$,

$$
\frac{\partial P_{\mathrm{MC}}}{\partial \theta_{j}}=\int_{-\infty}^{\infty} I(\mathbf{x}) \cdot \frac{\partial f_{\mathbf{x}}(\mathbf{x})}{\partial \theta_{j}} \cdot d \mathbf{x}+\mathrm{BT},
$$

where BT denotes a "boundary term" that arises if a perturbation in parameter $\theta_{j}$ affects the failure domain. This term is discussed in more detail below.

If the random variables are independent, the joint probability density function can be written as a product of the individual random variable probability density functions as

$$
f_{\mathbf{x}}(\mathbf{x})=\prod_{i=1}^{\mathrm{nrv}} f_{X_{i}}\left(x_{i}\right)
$$

where nrv denotes the number of random variables.

The parameter $\theta_{j}$ is a parameter of only one of the probability density functions comprising the joint density for the independent random variables, therefore, $\partial f_{\mathbf{x}}(\mathbf{x}) / \partial \theta_{j}=$ $\partial f_{X_{j}}\left(x_{j}\right) / \partial \theta_{j}$ and (12) becomes

$$
\frac{\partial P_{\mathrm{MC}}}{\partial \theta_{j}}=\int_{-\infty}^{\infty} I(\mathbf{x}) \cdot \frac{\partial f_{X}\left(x_{j}\right)}{\partial \theta_{j}} \cdot \frac{1}{f_{X_{j}}\left(x_{j}\right)} \cdot f_{x}(\mathbf{x}) \cdot d \mathbf{x}+\mathrm{BT} .
$$

The expected value operation in the above integral can be approximated using Monte Carlo sampling as

$$
\frac{\partial P_{\mathrm{MC}}}{\partial \theta_{j}} \approx \frac{1}{M} \sum_{i=1}^{M}\left[I\left(\mathbf{x}_{i}\right) \cdot \kappa\left(x_{j, i}, f_{X}, \theta_{j}\right)\right]+\mathrm{BT},
$$




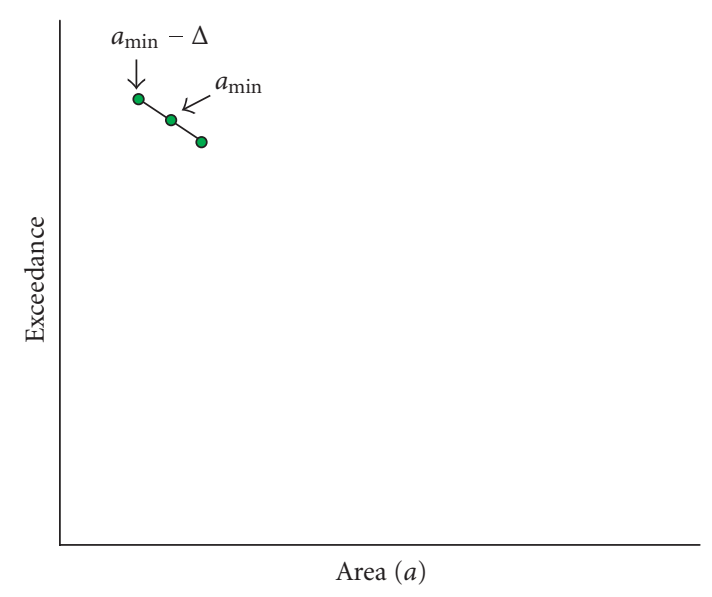

FIGURE 2: Illustration: perturbation of $a_{\text {min }}$.

where $M$ is the number of samples, $x_{j, i}$ is the ith sample of random variable $j$, and $\kappa$ is a kernel function defined generically as

$$
\kappa\left(x, f_{x}, \theta\right)=\frac{d f_{x}(x)}{d \theta} \cdot \frac{1}{f_{x}(x)}=\frac{d \ln \left[f_{x}(x)\right]}{d \theta}
$$

$\kappa$ is distribution and parameter specific and is derived analytically for each distribution type and parameter of interest.

\subsubsection{Sensitivity of $P_{\mathrm{MC}}$ with respect to median and cov of stress and propagation scatter}

The following derivation applies equally to the stress scatter and propagation scatter random variables because both variables are modeled with a lognormal distribution. The kernel functions for a lognormal distribution with respect to the median and cov are

$$
\begin{gathered}
\kappa(x, \log \text { normal, } \tilde{X})=\frac{\ln (x)-\ln (\tilde{X})}{\tilde{X} \cdot \ln \left(1+\operatorname{cov}^{2}\right)}, \\
\kappa(x, \log \text { normal, cov }) \\
=\frac{\operatorname{cov} \cdot\left(-\ln \left(1+\operatorname{cov}^{2}\right)+(\ln (\tilde{X})-\ln (x))^{2}\right)}{\left(1+\operatorname{cov}^{2}\right) \cdot \ln \left(1+\operatorname{cov}^{2}\right)^{2}} .
\end{gathered}
$$

The sensitivity of the conditional probability-of-fracture can be determined from (15) using the appropriate kernel and not including the boundary term. A boundary term is not needed because perturbations in the median and cov of a log normal distribution do not affect the integration domain.

\subsubsection{Sensitivity of $P_{\mathrm{MC}}$ with respect to $a_{\min }$}

The sensitivity with respect to $a_{\min }$ is defined such that the slope of the exceedance curve at $a_{\min }$ is unchanged, as shown in Figure 2. The kernel function with respect to $a_{\min }$ for a

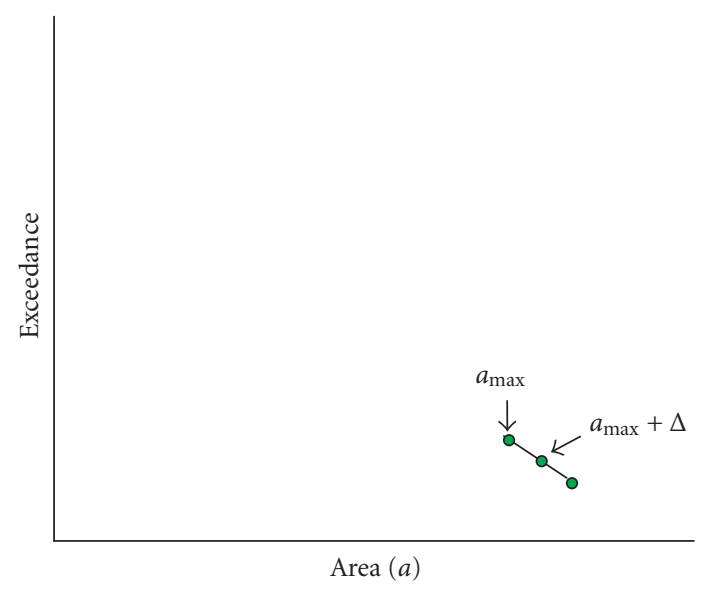

FIGURE 3: Illustration: perturbation of $a_{\max }$.

tabular exceedance curve is derived as

$$
\kappa\left(a, f_{A}, a_{\min }\right)=\frac{d f_{A}(a)}{d a_{\min }} \cdot \frac{1}{f_{A}\left(a_{\min }\right)}=f_{A}\left(a_{\min }\right),
$$

where $f_{A}\left(a_{\min }\right)$ is the pdf of the defect distribution evaluated at $a_{\min }$. The sensitivity is

$$
\frac{\partial P_{\mathrm{MC}}}{\partial a_{\mathrm{min}}}=E[I(\mathbf{x})] \cdot f_{A}\left(a_{\min }\right)+\mathrm{BT}=P_{\mathrm{MC}} \cdot f_{A}\left(a_{\min }\right)+\mathrm{BT} .
$$

$\mathrm{BT}$ is approximated as zero, see the appendix. Using this approximation, the sensitivity is

$$
\frac{\partial P_{\mathrm{MC}}}{\partial a_{\min }}=P_{\mathrm{MC}} \cdot f_{A}\left(a_{\mathrm{min}}\right)
$$

Equation (21) provides an upper bound to the true sensitivity.

\subsubsection{Sensitivity of $P_{\mathrm{MC}}$ with respect to $a_{\max }$}

The sensitivity with respect to $a_{\max }$ is defined such that the slope of the exceedance curve at $a_{\max }$ is unchanged, as shown in Figure 3.

The kernel function with respect to $a_{\max }$ for a tabular exceedance curve is derived as

$$
\kappa\left(a, f_{A}, a_{\max }\right)=\frac{d f_{A}(a)}{d a_{\max }} \cdot \frac{1}{f_{A}(a)}=-f_{A}\left(a_{\max }\right),
$$

where $f_{A}\left(a_{\max }\right)$ is the pdf of the defect distribution evaluated at $a_{\max }$ and the sensitivity is

$$
\frac{\partial P_{\mathrm{MC}}}{\partial a_{\max }}=-E[I(\mathbf{x})] \cdot f_{A}\left(a_{\max }\right)+\mathrm{BT}=-P_{\mathrm{MC}} \cdot f_{A}\left(a_{\max }\right)+\mathrm{BT} .
$$

BT is approximated as $f_{A}\left(a_{\max }\right)$, see the appendix. Using this approximation, the sensitivity becomes

$$
\frac{\partial P_{\mathrm{MC}}}{\partial a_{\max }}=f_{A}\left(a_{\max }\right)\left(1-P_{\mathrm{MC}}\right) .
$$

This sensitivity is an upper bound to the true sensitivity. 


\subsection{Variance estimates}

The sensitivity values are estimated using Monte Carlo sampling, therefore the sensitivities themselves are random variables. As such, variance estimates and confidence bounds are needed. The 95\% confidence bounds are then computed assuming the variation follows a normal distribution as

$$
S_{\theta}-1.96 \sqrt{V} \leq S_{\theta} \leq S_{\theta}+1.96 \sqrt{V},
$$

where $S_{\theta}$ denotes the sensitivity of interest.

\subsubsection{Variance estimate of sensitivity equation: zone-based conditional POF}

The variance estimate of the conditional POF per zone sensitivity is derived below. Different equations are used for the variance of $a_{\min }$ and $a_{\max }$ than for propagation and stress scatter since simpler equations can be developed.

\subsubsection{Stress and propagation scatter}

$$
V\left[\frac{\partial P_{\mathrm{MC}}}{\partial \theta}\right]=\frac{1}{M^{2}} \sum_{k=1}^{M}\left(I(\mathbf{x}) \frac{\partial f}{\partial \theta} \cdot \frac{1}{f}\right)^{2}-\frac{S_{\theta}^{2}}{M} .
$$

\subsection{3. $a_{\min }$ and $a_{\max }$.}

The equation for $\partial P_{\mathrm{MC}} / \partial \theta$ simplifies for $\theta$ equal to $a_{\min }$ or $a_{\max }$. This information can be used to develop a simplified variance equation as follows:

$$
V\left[\frac{\partial P_{\mathrm{MC}}}{\partial a_{\min }}\right]=f_{A}^{2}\left(a_{\mathrm{min}}\right) V\left[P_{\mathrm{MC}}\right]=f_{A}^{2}\left(a_{\mathrm{min}}\right) \frac{P_{\mathrm{MC}}\left(1-P_{\mathrm{MC}}\right)}{M}
$$

since $V\left[P_{\mathrm{MC}}\right]$ is obtained from standard sampling estimates [27] as $V\left[P_{\mathrm{MC}}\right]=\left(\left(1-P_{\mathrm{MC}}\right) P_{\mathrm{MC}}\right) / M$.

Similarly,

$$
V\left[\frac{\partial P_{\mathrm{MC}}}{\partial a_{\max }}\right]=f_{A}^{2}\left(a_{\max }\right) \frac{P_{\mathrm{MC}}\left(1-P_{\mathrm{MC}}\right)}{M} .
$$

\subsubsection{Variance estimate: zone-based unconditional POF}

The variance of the zone-based unconditional POF sensitivity can be derived from the conditional POF as follows:

$$
V\left[\frac{\partial P_{F, i}}{\partial \theta}\right]=\left(1-P_{F, i}\right)^{2}\left(\left(\frac{\partial \lambda_{i}}{\partial \theta}\right)^{2} V\left[P_{\mathrm{MC}_{i}}\right]+\lambda_{i}^{2} V\left[\frac{\partial P_{\mathrm{MC}, i}}{\partial \theta}\right]\right),
$$

where the term $\partial \lambda_{i} / \partial \theta$ is only nonzero when formulating the sensitivity with respect to $a_{\mathrm{min}}$ of the exceedance curve.

For $a_{\min }$, (29) can be expanded and simplified as

$$
\begin{aligned}
V\left(\frac{\partial P_{F, i}}{\partial a_{\min }}\right)= & \left(\left(1-P_{F, i}\right) \cdot \lambda_{i} \cdot f_{A}\left(a_{\min }\right) \frac{N\left(a_{\max }\right)}{N\left(a_{\min }\right)}\right)^{2} \\
& \cdot \frac{P_{\mathrm{MC}_{i}} \cdot\left(1-P_{\mathrm{MC}_{i}}\right)}{M_{i}},
\end{aligned}
$$

where $M_{i}$ indicates the number of samples used for zone $i$.

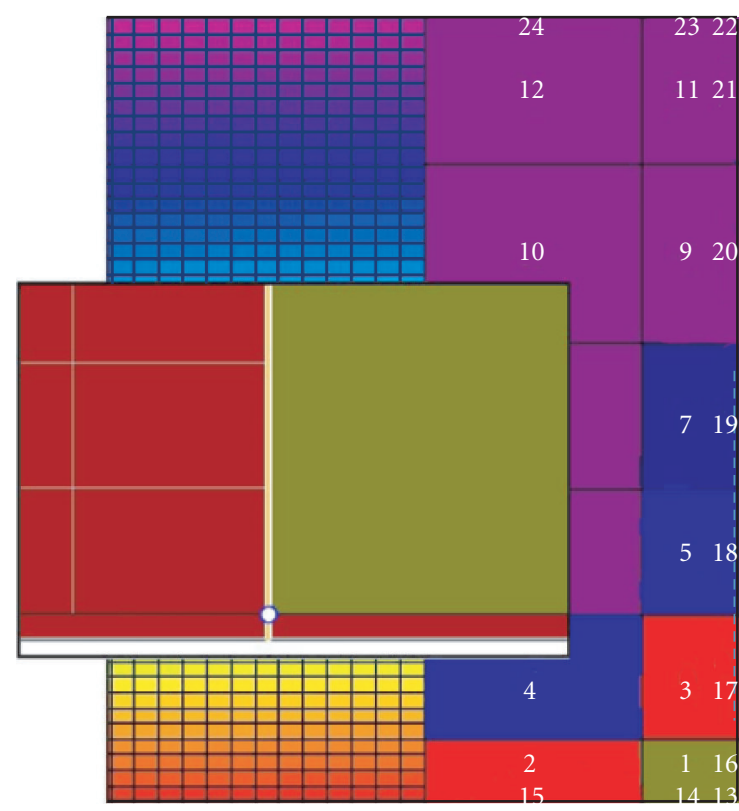

FIGURE 4: Zone discretization of rectangular cross-section with blowup of zone 2 .

\subsubsection{Variance estimates: disk POF}

The variance estimates for the disk can be derived from (7) as

$$
V\left[\frac{\partial P_{F}}{\partial \theta}\right]=\left(1-P_{F}\right)^{2} \sum_{i=1}^{\hat{m}} \frac{1}{\left(1-P_{F, i}\right)^{2}} V\left[\frac{\partial P_{F, i}}{\partial \theta}\right] .
$$

\subsection{Computational procedure}

The sequence of analysis becomes as follows: (a) for each zone, determine the conditional POF $\left(P_{\mathrm{MC}}\right)$ using $(11)$, and the sensitivity of the conditional $\mathrm{POF}\left(\partial P_{\mathrm{MC}} / \partial \theta\right)$ using, generically, (15), (b) determine the unconditional POF for each zone from (1), and the sensitivity of the unconditional POF using (8), (c) determine the POF for the disk using (2), and the sensitivity of the disk POF using (7), (d) determine variance estimates and confidence bounds for the sensitivities using ((25), (26)-(28), (25)-(31)).

\section{NUMERICAL EXAMPLE}

The model consists of a titanium ring as outlined by advisory circular AC-33.14-1 [13]. The titanium ring is axisymmetric, has a rectangular cross-section, is under rotation, and has an external load applied at the rim to simulate blade loading. The maximum principle stress is in the circumferential direction. The crack grows under constant amplitude cyclical loading, for example, startup-shutdown, until fracture, defined as when the stress intensity factor exceeds the fracture toughness. The probability-of-fracture of the disk before twenty thousand cycles is computed using Monte Carlo sampling.

The titanium ring cross-section has been divided into 24 zones as shown in Figure 4. Only half the cross-section has 
TABLE 1: Example problem input data.

\begin{tabular}{|c|c|c|}
\hline Property & Description & Comment \\
\hline Loading & $\begin{array}{l}\text { Constant amplitude, } 0-81-0 \mathrm{ksi} \\
\qquad(558 \mathrm{MPa})\end{array}$ & Stress due to RPM-max speed $=6800 \mathrm{RPM}$ \\
\hline $\mathrm{da} / \mathrm{dN}-\Delta \mathrm{K}$ & $\begin{aligned} \mathrm{C}= & 5.248 \mathrm{E}-11(9.25 \mathrm{E}-13 \mathrm{SI}) \\
\mathrm{m}= & 3.87, \mathrm{Kc}=58.7 \mathrm{ksi}-\mathrm{in}^{\wedge}-1 / 2 \\
& \left(64.6 \mathrm{MPa}-\mathrm{m}^{\wedge}-1 / 2\right)\end{aligned}$ & $\begin{array}{l}\text { Paris law with no threshold } \\
\text { (C-constant, m-exponent, Kc-fracture toughness) } \\
\text { US-units: da/dN (in/cycle), } \Delta \mathrm{K}\left(\mathrm{ksi}^{-} \mathrm{in}^{\wedge}-1 / 2\right) \\
\text { SI-units: } \mathrm{da} / \mathrm{dN}(\mathrm{m} / \text { cycle }), \Delta \mathrm{K}\left(\mathrm{MP}_{a}-\mathrm{m}^{\wedge}-1 / 2\right)\end{array}$ \\
\hline Initial crack size & aia.post $95.3 \mathrm{fbh} .3 \mathrm{fbh} . \mathrm{dist}$ & $\begin{array}{l}\text { Post } 95 \text { material, inspected in billet and forging } \\
\text { with a \#3 flat bottom hole ultrasonic inspection }\end{array}$ \\
\hline Propagation scatter & Median $=1, \operatorname{cov}=0.1$ & $\log$ normal distribution—for all zones \\
\hline stress scatter & Median $=1, \operatorname{cov}=0.1$ & log normal distribution \\
\hline
\end{tabular}

been modeled due to symmetry. The stresses are determined from finite element analysis. A blowup of zone 2 is shown in the inset. The initiation site of the defect is located at the life limiting location of the zone-demarcated with a white circle in Figure 4. In practice, a zone-refinement convergence algorithm [28] would ordinarily be exercised in order to ensure accuracy but is not germane for the purpose of verifying the sensitivity equations. The exceedance curve used to represent the initial crack size distribution is obtained from industrydefined data [6] and represents rotor-grade titanium that has been nondestructively inspected in the billet and forging stages by an ultrasonic inspection method calibrated to a \#3 flat bottom hole. The aspect ratio of the initial crack is one (the crack may become elliptical in shape during crack growth depending upon the stress gradient and proximity to a free surface). The input data summarizing the problem are given in Table 1.

The numerical results from the sensitivity equations are compared with finite difference estimates. The finite difference estimates are obtained by perturbing the parameter of interest, rerunning the analysis, and approximating the sensitivity as

$$
\frac{\partial P}{\partial \theta} \approx \frac{\Delta P}{\Delta \theta}=\frac{P(\theta+\Delta \theta)-P(\theta)}{\Delta \theta},
$$

where $P$ represents the POF (either zone (conditional or unconditional) or the disk) and $\Delta \theta$ represents the perturbed parameter. The perturbation size is chosen arbitrarily as a few percent of nominal. The perturbation size must be large enough to evoke a significant enough change in the computed POF relative to the unperturbed analysis.

We note that it is exceedingly laborious to obtain finite difference estimates in that a very large number of Monte Carlo samples must be used in order to prevent the difference in the POF from being masked by sampling variance, or a very refined importance sampling method must be used $[1,29]$. In addition, a separate analysis must be performed for each sensitivity estimate. In the examples below, six additional analyses are required to obtain the finite difference estimates, whereas, the sensitivity equations provide all sensitivities in a single analysis.
TABLE 2: Comparison of conditional POF sensitivity equations and finite difference estimates.

\begin{tabular}{llc}
\hline $\begin{array}{l}\text { Random } \\
\text { variable parameter }\end{array}$ & $\begin{array}{l}\text { Sensitivity } \\
\text { method }\end{array}$ & $\begin{array}{l}\text { Finite } \\
\text { difference }\end{array}$ \\
\hline $\begin{array}{l}\text { Propagation scatter } \\
\text { median }\end{array}$ & $-5.148 \mathrm{E}-2$ & $-5.195 \mathrm{E}-2$ \\
\hline $\begin{array}{l}\text { Propagation scatter } \\
\text { cov }\end{array}$ & $4.765 \mathrm{E}-3$ & $5.500 \mathrm{E}-3$ \\
\hline $\begin{array}{l}\text { Stress scatter } \\
\text { median }\end{array}$ & $2.037 \mathrm{E}+2$ & $2.093 \mathrm{E}+2$ \\
\hline $\begin{array}{l}\text { Stress scatter } \\
\text { cov }\end{array}$ & $9.042 \mathrm{E}-2$ & $9.860 \mathrm{E}-2$ \\
\hline $\begin{array}{l}\text { Exceedance curve } \\
a_{\text {min }}\end{array}$ & $6.497 \mathrm{E}-3$ & $6.416 \mathrm{E}-3$ \\
\hline $\begin{array}{l}\text { Exceedance curve } \\
a_{\text {max }}\end{array}$ & $3.260 \mathrm{E}-10$ & $3.602 \mathrm{E}-10$ \\
\hline
\end{tabular}

a2E6 samples used for all analyses.

\subsection{Single zone verification: conditional POF}

In this example, only Zone 2, see inset Figure 4, is considered. Zone 2 is a principal zone in that a large portion of the disk risk originates from the probability of a defect being contained in the zone and growing to fracture; primarily due to the high stress state and the near surface effect.

Table 2 shows the comparison of the sensitivity $\partial P_{\mathrm{MC}} / \partial \theta$ with respect to finite difference approximations. Two million samples were used for all Monte Carlo analyses in order to minimize sampling variance. The perturbations sizes used were one percent for propagation scatter median and cov and stress scatter median, and five percent perturbations were used for the stress scatter cov and the bounds of the exceedance curve. The perturbations sizes were chosen to be of size such that the change in the POF was sufficient to calculate finite difference estimates.

The comparisons between the equations and finite difference estimates are very close. A positive sensitivity indicates 
TABLE 3: Comparison of variation of sensitivity estimates equation versus statistical trial-stress scatter random variable.

\begin{tabular}{ccccc}
\hline $\begin{array}{c}\text { Stress scatter } \\
\mathrm{M}=10000\end{array}$ & $\begin{array}{c}S_{\tilde{X}} \\
\text { equation }\end{array}$ & $\begin{array}{c}S_{\tilde{X}} \\
\text { sampling }\end{array}$ & $\begin{array}{c}S_{\text {cov }} \\
\text { equation }\end{array}$ & $\begin{array}{c}S_{\text {cov }} \\
\text { sampling }\end{array}$ \\
\hline Mean & $8.05 \mathrm{E}-02$ & $8.80 \mathrm{E}-02$ & $6.32 \mathrm{E}-2$ & $7.83 \mathrm{E}-02$ \\
\hline $\begin{array}{c}\text { Standard } \\
\text { deviation }\end{array}$ & $1.22 \mathrm{E}-2$ & $1.25 \mathrm{E}-02$ & $2.13 \mathrm{E}-2$ & $2.14 \mathrm{E}-02$ \\
\hline cov & $15.1 \%$ & $14.2 \%$ & $33.7 \%$ & $27.3 \%$ \\
\hline $\begin{array}{c}\text { Lower 95\% } \\
\text { conf limit }\end{array}$ & $5.66 \mathrm{E}-02$ & $6.29 \mathrm{E}-02$ & $2.11 \mathrm{E}-02$ & $3.56 \mathrm{E}-02$ \\
\hline $\begin{array}{c}\text { Upper 95\% } \\
\text { conf limit }\end{array}$ & $1.04 \mathrm{E}-01$ & $1.13 \mathrm{E}-01$ & $1.05 \mathrm{E}-01$ & $1.21 \mathrm{E}-01$ \\
\hline
\end{tabular}

${ }^{\mathrm{e} B a s e d}$ on 3000 statistical trials, 10000 Monte Carlo samples in each trial.

that an increase in the parameter will increase the POF, and conversely, a negative sensitivity indicates that an increase in the parameter will decrease the POF. The propagation scatter median sensitivity is negative because this variable multiplies the computed cycles-to-failure estimated from fracture mechanics algorithms. Thus, a larger value increases the cyclesto-failure and, therefore, reduces the POF.

\subsubsection{Variance equations}

The variance equations are verified by comparing the predicted variance against the results from a statistical experiment. The statistical experiment is as follows. For a fixed number of samples within a zone, for example, 10000 , compute the sensitivity estimates given a particular random number seed; modify the seed and repeat. The statistical results of the sensitivity estimates can be used to estimate the sampling population of the sensitivities and the corresponding mean, variance, and $95 \%$ confidence limits of the estimate. For a sufficiently large number of statistical trials, these results will converge to the true mean, variance, and so forth, and can be used to compare against the equation approximations. Of course the results from the equations are obtained from only a single analysis whereas the statistical trials require hundreds or thousands of runs.

Table 3 shows the comparison of the mean, standard deviation, coefficient of variation, and $95 \%$ confidence bounds of the stress scatter sensitivity estimates and variance estimates from equations versus the statistical trials for the case of ten thousand Monte Carlo samples used in each trial. The statistical results are based on three thousand trials. Table 4 shows the coefficient of variation results from equations and sampling as a function of the number of samples used in each trial. The results indicate that the variance equations agree well with the statistical trials. The very small variation in $a_{\max }$ results because the boundary term, $f_{A}\left(a_{\max }\right)$, is not affected by sampling variance and this term dominates the sensitivity estimate.

Figure 5 shows a normal probability plot of the stress scatter median results from three thousand statistical trials with 10000 samples in each trial. The results clearly indicate that the variance of the sensitivity estimate follows a normal distribution. Other sensitivity estimates have a similar behavior.

Figure 6 shows the behavior of the variance of the stress scatter median as a function of the number of samples, indicating that the variance of the sensitivity estimate is reduced as the reciprocal of the number of samples. Other sensitivity measures behave similarly.

\subsection{Multizone, 24 zones total}

A comprehensive analysis considering all twenty-four zones is considered next. The finite difference results were obtained as before except importance sampling $[1,29]$ which was used to compute the POF for the finite difference estimates instead of Monte Carlo sampling in order to reduce run-time.

The results are shown in Table 5. The equations and the finite difference results are in good agreement. A "cumulative" sensitivity for the disk for propagation scatter median and cov is computed by summing over all zones in (7). The sensitivity with respect to $a_{\min }$ shows some disparity due to the extremely small sensitivity.

\section{CONCLUSIONS}

A methodology is developed and demonstrated that determines the sensitivities of the probability-of-fracture of a zone-based probabilistic fatigue analysis with respect to the parameters of the probability distributions of the input random variables without additional fatigue calculations. The methodology is demonstrated using a probabilistic fatigue analysis of a gas turbine compressor disk and verified against finite difference estimates. The elements of the methodology include the following:

(i) sensitivities are computed at the zone level (both conditional and unconditional) and for the entire disk,

(ii) sensitivities are computed with respect to the median and cov of the crack propagation and stress scatter random variables and with respect to the bounds of the initial crack size exceedance curve,

(iii) the necessity for the boundary term was discussed and limiting solutions were obtained for the bounds of the initial crack size exceedance curve,

(iv) variance estimates are developed and used to determine upper and lower $95 \%$ confidence bounds on the sensitivity estimates,

(v) the formulas use the existing failure samples and no further fatigue calculations are required,

(vi) the methodology can be extended to consider other random variables and distribution functions.

We note that finite difference estimates of the probabilityof-fracture, although theoretically possible, are extremely laborious and involve multiple analyses with very high precision requirements on the probability calculations. Therefore, a mathematical development and corresponding software implementation is necessary to make such sensitivity calculations practical. 
TABLE 4: Comparison of coefficient of variation, in percent, as a function of number of samples per zone.

\begin{tabular}{ccccccc}
\hline \# Samples & SS median & SS cov & PS median & PS cov & $a_{\min }$ & $a_{\max }$ \\
\hline $2500^{\mathrm{f}}$ & $24.0 / 28.4$ & $37.5 / 55.9$ & $77.6 / 83.5$ & $735 / 538$ & $20.8 / 22.1$ & $0.20 / 0.18$ \\
\hline $10000^{\mathrm{g}}$ & $15.3 / 14.2$ & $24.5 / 27.3$ & $39.2 / 41.1$ & $112 / 229$ & $10.2 / 11.1$ & $0.10 / 0.09$ \\
\hline $50000^{\mathrm{h}}$ & $6.4 / 6.3$ & $12.4 / 12.2$ & $19.6 / 18.2$ & $55 / 121$ & $4.9 / 4.9$ & $0.04 / 0.04$ \\
\hline $100000^{\mathrm{i}}$ & $4.5 / 4.8$ & $9.5 / 8.3$ & $10.8 / 11.2$ & $69 / 104$ & $3.5 / 3.2$ & $0.03 / 0.03$ \\
\hline $500000^{\mathrm{i}}$ & $4.9 / 2.0$ & $8.3 / 3.7$ & $11.2 / 6.2$ & $40 / 105$ & $1.6 / 3.2$ \\
\hline
\end{tabular}

${ }^{\mathrm{f}} 10000$ statistical trials, ${ }^{\mathrm{g}} 3000$ statistical trials, ${ }^{\mathrm{h}} 300$ statistical trials, ${ }^{\mathrm{i}} 100$ statistical trials.

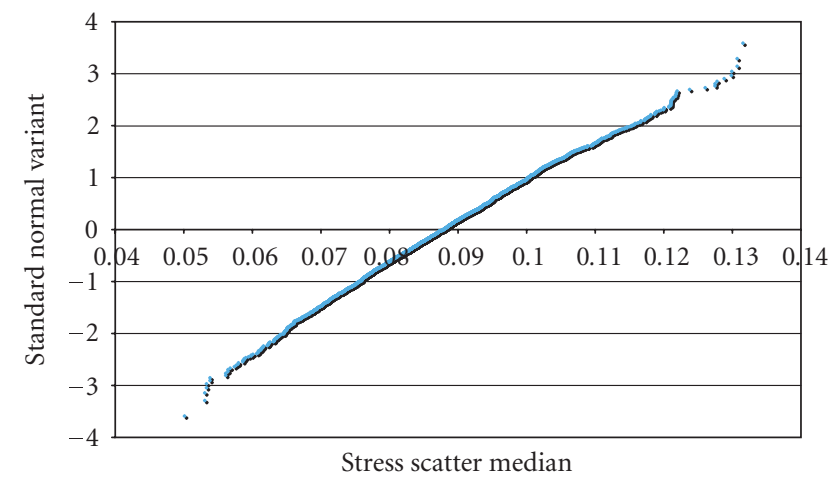

FIGURE 5: Normal probability plot of stress scatter sensitivity estimates (3000 statistical trials).

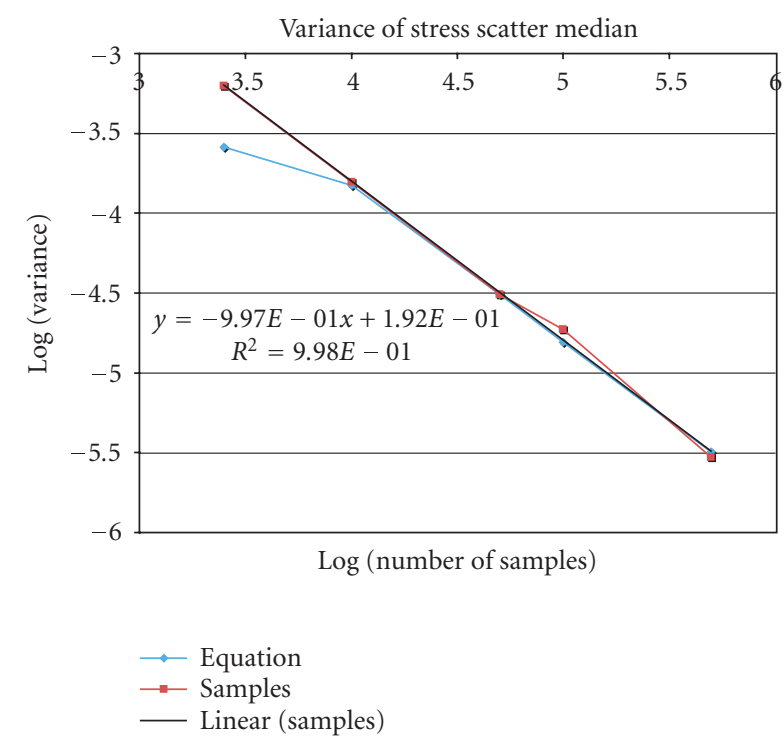

FIgURE 6: Variance of stress scatter median sensitivity estimate on log-log scale.

\section{APPENDIX}

\section{BOUNDARY TERM DERIVATION}

A boundary term will be needed if the derivative is being taken with respect to a parameter that explicitly affects the
TABLe 5: Comparison of disk POF sensitivity equations with finite difference estimates.

\begin{tabular}{ccc}
\hline $\begin{array}{c}\text { Random } \\
\text { variable parameter }\end{array}$ & $\begin{array}{c}\text { Sensitivity } \\
\text { method }\end{array}$ & $\begin{array}{c}\text { Finite } \\
\text { difference }^{\mathrm{d}}\end{array}$ \\
\hline $\begin{array}{c}\text { Stress scatter } \\
\text { median }\end{array}$ & $1.035 \mathrm{E}+0$ & $1.066 \mathrm{E}+0$ \\
\hline $\begin{array}{c}\text { Stress scatter } \\
\text { cov }\end{array}$ & $7.409 \mathrm{E}-4$ & $7.796 \mathrm{E}-4$ \\
\hline $\begin{array}{c}\text { Exceedance curve } \\
a_{\text {min }}\end{array}$ & $8.883 \mathrm{E}-9$ & $6.580 \mathrm{E}-8$ \\
\hline $\begin{array}{c}\text { Exceedance curve } \\
a_{\text {max }}\end{array}$ & $5.989 \mathrm{E}-12$ & $5.258 \mathrm{E}-12$ \\
\hline $\begin{array}{l}\text { Propagation scatter } \\
\text { median-cumulative }\end{array}$ & $-2.369 \mathrm{E}-4$ & $-2.553 \mathrm{E}-4$ \\
\hline $\begin{array}{c}\text { Propagation scatter } \\
\text { cov-cumulative }\end{array}$ & $5.891 \mathrm{E}-5$ & $4.938 \mathrm{E}-5$ \\
\hline
\end{tabular}

${ }^{\mathrm{d}}$ Importance sampling $[1,29]$.

failure domain. Many situations do not need the boundary term. For example, derivatives of a normal distribution with respect to the mean or standard deviation, or derivatives of a log normal distribution with respect to the median or cov do not require a boundary term. Examples where a boundary term is required are derivatives with respect to the bounds of any truncated distribution such as a uniform, truncated normal, or a tabular exceedance curve. In this research, sensitivities with respect to the bounds of the anomaly exceedance curve, $a_{\min }$ and $a_{\max }$, require consideration of a boundary term.

For one random variable problems Leibnitz's rule is used to determine the boundary term:

$$
\begin{aligned}
\frac{d P}{d \theta}= & \frac{d}{d \theta} \int_{a(\theta)}^{b(\theta)} f_{x}(x) d x=\int_{a(\theta)}^{b(\theta)} \frac{d f_{x}(x)}{d \theta_{j}} d x+f(b(\theta), x) \\
& \cdot \frac{d b(\theta)}{d \theta}-f(a(\theta), x) \cdot \frac{d a(\theta)}{d \theta} .
\end{aligned}
$$


For fatigue analysis, failure is defined as the integral from $a^{*}$ (the initial crack size that will just cause failure) to $a_{\max }$ :

$$
\begin{aligned}
\frac{\partial P_{\mathrm{MC}}}{\partial a_{\max / \min }}= & \frac{\partial}{\partial a_{\max / \min }} \int_{a^{*}}^{a_{\max }} f_{a}(a) d a \\
= & \int_{a^{*}}^{a_{\max }} \frac{\partial f_{a}(a)}{\partial a_{\max / \min }} d a+\frac{d a_{\max }}{d a_{\max / \min }} f\left(a_{\max }\right) \\
& -\frac{d a^{*}}{d a_{\max / \min }} f\left(a_{\max }\right),
\end{aligned}
$$

where $a_{\max } / \min$ stands for either $a_{\min }$ or $a_{\max }$.

The derivatives become

$$
\frac{d a_{\max }}{d a_{\max }}=1, \quad \frac{d a_{\max }}{d a_{\max }}=0, \quad \frac{d a^{*}}{d a_{\min }}=0, \quad \frac{d a^{*}}{d a_{\max }}=0
$$

and the boundary terms for $a_{\min }$ and $a_{\max }$ are

$$
\mathrm{BT}_{a_{\min }}=0, \quad \mathrm{BT}_{a_{\max }}=f\left(a_{\max }\right) .
$$

If the crack size is the only random variable, the boundary term with respect to $a_{\min }$ is zero. However, for higherdimensional problems, that is, more random variables, a portion of the failure domain could intersect the situation where $x_{1}=a_{\min }$ and a boundary term would be nonzero. In this situation, calculation of the boundary term cannot be obtained without additional sampling. It can be shown that the boundary term is related to $f_{A}\left(a_{\min }\right) P\left[g(\mathbf{x}) \leq 0 \mid x_{1}=a_{\min }\right]$. That is, we solve a probability problem with the initial crack size set to $a_{\mathrm{min}}$. However, for most problems the boundary term will be close to zero because $P\left[g(\mathbf{x}) \leq 0 \mid x_{1}=a_{\text {min }}\right]$ will likely be small if not zero. Therefore, by default, we assume the boundary term for sensitivities with respect to $a_{\text {min }}$ is zero. This sensitivity estimate is an upper bound to the true sensitivity.

If the crack size is the only random variable, the boundary term with respect to $a_{\max }$ is $f_{A}\left(a_{\max }\right)$. However, for higher-dimensional problems, however, a portion of the failure domain may intersect the condition where $x_{1}=a_{\max }$ and the boundary term may not be equal to $f_{A}\left(a_{\max }\right)$. In this situation, calculation of the boundary term cannot be obtained without additional computation. It can be shown that boundary term is related to $f_{A}\left(a_{\max }\right) P\left[g(\mathbf{x}) \leq 0 \mid x_{1}=a_{\max }\right]$. Thus, the estimate $f_{A}\left(a_{\min }\right)$ is an upper bound to the true boundary term for problems with additional random variables.

For most problems, the boundary term will be close to $f_{A}\left(a_{\max }\right)$ because $P\left[g(\mathbf{x}) \leq 0 \mid x_{1}=a_{\max }\right]$ will likely be close to one. That is, the conditional POF given that all cracks initiate at the largest size of the exceedance curve will be close to one. Therefore, by default, we include the boundary term for sensitivities with respect to $a_{\max }$ and use a value equal to $f_{A}\left(a_{\max }\right)$.

\section{ACKNOWLEDGMENTS}

This work was supported in part by the Federal Aviation Administration under Cooperative Agreement 95-G-041 and
Grant 99-G-016. The authors wish to thank the FAA Technical Center project managers, Bruce Fenton and Joe Wilson for their continued diligence and encouragement, Tim Mouzakis of the FAA Engine and Propeller Directorate for his continued support, and Drs. Craig McClung, Michael Enright, and Luc Huyse of Southwest Research Institute for providing leadership and guidance. The ongoing contributions of the project Steering Committee Members from Honeywell, General Electric, Pratt \& Whitney, and Rolls-Royce are also gratefully acknowledged. This work was also supported in part under Air Force Agreement No. F33615-032-5203, Dr. Patrick J. Golden, AFRL/MLLMN, Project Monitor, and a subcontract with Southwest Research Institute, Dr. Stephen Hudak Jr., Program Manager. The authors would also like to acknowledge the assistance of Dr. James Larsen, AFRL/MLLMN, and the high performance computer facilities at Wright-Patterson AFB, Dayton, OH.

\section{NOMENCLATURE}

$$
\begin{aligned}
& \text { a } \quad=\text { defect size } \\
& =\text { minimum defect size of the initial crack size } \\
& \text { exceedance curve } \\
& =\text { maximum defect size of the initial crack size } \\
& \text { exceedance curve } \\
& =\text { random variable representing uncertainty in } \\
& \text { fatigue crack propagation scatter } \\
& \text { cov }=\text { coefficient of variation } \\
& E[\cdot] \quad=\text { expected value operator } \\
& f_{x}(\mathbf{x}) \quad=\text { joint probability density function } \\
& \text { of vector of random variables } \mathbf{x} \\
& f_{A}(a) \quad=\text { probability density function of the initial } \\
& \text { crack size exceedance curve } \\
& =\text { probability density function of the initial } \\
& f_{A}\left(a_{\min }\right) \quad \text { crack size exceedance curve } \\
& \text { evaluated at } a_{\min } \\
& =\text { probability density function of the initial } \\
& f_{A}\left(a_{\max }\right) \quad \text { crack size exceedance } \\
& \text { curve evaluated at } a_{\max } \\
& g(\mathbf{x}) \quad=\text { limit state function } \\
& I(\mathbf{x}) \quad=\text { indicator function } \\
& m \quad \text { = number of zones } \\
& \hat{m} \quad=\text { number of zones affected by parameter } \theta \\
& M \quad=\text { number of samples } \\
& \text { nrv }=\text { number of random variables } \\
& N(a) \quad=\text { exceedance value associated with } \\
& \text { initial crack size } \\
& \text { = cycles-to-failure used to compute } \\
& \text { probability-of-fracture } \\
& \text { = cycles-to-failure predicted by } \\
& \text { fracture mechanics algorithm }
\end{aligned}
$$

$N_{f} \quad$ cycles-to-failure used to compute
probability-of-fracture
$N_{f}^{\prime} \quad \begin{gathered}\text { cycles-to-failure predicted by } \\ \text { fracture mechanics algorithm }\end{gathered}$




$$
\begin{aligned}
& P \quad=\text { probability-of-fracture } \\
& P_{\mathrm{MC}}=\text { conditional probability-of-fracture } \\
& \text { computed from Monte Carlo sampling } \\
& =\text { probability-of-fracture for a defect } \\
& \text { originating within zone } k \\
& P_{F} \quad=\text { probability-of-fracture of a disk } \\
& \text { = random variable representing uncertainty } \\
& \text { in applied loading } \\
& V[\cdot]=\text { variance operator } \\
& \tilde{X} \quad=\text { median value of random variable } X \\
& =\text { vector of random variables, } \\
& \mathbf{x}=\left(x_{1}, x_{2}, \ldots, x_{\text {nrv }}\right) \\
& =\text { kernel function for determining } \\
& \text { sampling-based sensitivities } \\
& \lambda=\text { mean anomaly occurrence rate } \\
& \theta_{j} \quad=\text { statistical parameter of probability density } \\
& \text { function associated with variable } x_{j} \\
& =\text { applied stress used to } \\
& \text { calculate probability-of-fracture } \\
& \sigma^{\prime} \quad=\text { applied stress input from external source } \\
& =\text { reference volume or surface area of } \\
& \Psi \quad \text { component per initial crack size } \\
& \text { exceedance curve } \\
& \Psi_{i} \quad=\text { volume or surface area of zone }
\end{aligned}
$$

\section{REFERENCES}

[1] Y.-T. Wu, M. P. Enright, and H. R. Millwater, "Probabilistic methods for design assessment of reliability with inspection," AIAA Journal, vol. 40, no. 5, pp. 937-946, 2002.

[2] M. P. Enright and L. Huyse, "Methodology for probabilistic life prediction of multiple-anomaly materials," AIAA Journal, vol. 44, no. 4, pp. 787-793, 2006.

[3] M. P. Enright, L. Huyse, and R. C. McClung, "Fracture mechanics-based probabilistic life prediction of components with large numbers of inherent material anomalies," in Proceedings of the 9th International Conference on Structural Safety and Reliability (ICOSSAR '05), pp. 601-607, Rome, Italy, June 2005.

[4] G. R. Leverant, H. R. Millwater, R. C. McClung, and M. P. Enright, "A new tool for design and certification of aircraft turbine rotors," Journal of Engineering for Gas Turbines and Power, vol. 126, no. 1, pp. 155-159, 2004.

[5] H. R. Millwater, S. H. K. Fitch, Y.-T. Wu, et al., "A probabilistically-based damage tolerance analysis computer program for hard alpha anomalies in titanium rotors," in Proceedings of ASME International Gas Turbine \& Aeroengine Congress (IGTI), Munich, Germany, May 2000, ASME paper no. 2000-GT-0421.

[6] Aerospace Industries Association Rotor Integrity SubCommittee, "The development of anomaly distributions for aircraft engine titanium disk alloys," in Proceedings of 38th AIAA/ASME/ASCE/AHS/ASC Structures, Structural Dynamics, and Materials Conference, pp. 2543-2553, Orlando, Fla, USA, April 1997.
[7] G. R. Leverant, H. R. Millwater, D. L. Littlefield, Y.-T. Wu, and R. C. McClung, "A probabilistic approach to aircraft turbine rotor material design," in Proceedings of ASME International Gas Turbine \& Aeroengine Congress (IGTI), Orlando, Fla, USA, 1997, paper $97-G T-22$.

[8] G. R. Leverant, R. C. McClung, Y.-T. Wu, et al., "Turbine rotor material design," Final Report DOT/FAA/AR-00/64, Federal Aviation Administration, Washington, DC, USA, 2000.

[9] P. C. McKeighan, L. C. Perocchi, A. E. Nicholls, and R. C. McClung, "Characterizing the cracking behavior of hard alpha defects in rotor grade Ti-6-4 alloy," in Fatigue Behavior of Titanium Alloys, R. Boyer, D. Eylon, and G. Lutjering, Eds., pp. 211-218, The Minerals, Metals \& Materials Society, Warrendale, Pa, USA, 1999.

[10] P. J. Laz, K. S. Chan, R. C. McClung, and G. R. Leverant, "Effects of CTE-induced residual stresses around hard alpha particles on fatigue crack growth in Ti-6Al-4V," Fatigue and Fracture of Engineering Materials and Structures, vol. 26, no. 12, pp. 1145-1157, 2003.

[11] R. C. McClung, M. P. Enright, H. R. Millwater, G. R. Leverant, and S. J. Hudak Jr., "A software framework for probabilistic fatigue life assessment of gas turbine engine rotors," Journal of ASTM International, vol. 1, no. 8, Article ID JAI19025, 12 pages, 2004.

[12] R. C. McClung, M. P. Enright, Y.-D. Lee, and L. Huyse, "Efficient fracture design for complex turbine engine components," in Proceedings of ASME International Gas Turbine \& Aeroengine Congress (IGTI), Vienna, Austria, June 2004, ASME paper no. GT2004-53323.

[13] Federal Aviation Administration, "Advisory circular-damage tolerance for high energy turbine engine rotors," U.S. Department of Transportation, AC 33.14-1, 2001.

[14] M. P. Enright, Y.-D. Lee, R. C. McClung, et al., "Probabilistic surface damage tolerance assessment of aircraft turbine rotors," in Proceedings of the 48th ASME International Gas Turbine \& Aeroengine Technical Congress (IGTI), vol. 3, pp. 625-631, Atlanta, Ga, USA, June 2003, ASME paper GT200338731 .

[15] K. S. Chan, M. P. Enright, and J. Kong, "MicroFaVa: a micromechanical code for predicting fatigue life variability," in Material Damage Prognosis, J. M. Larsen, L. Christodoulou, J. R. Calcaterra, et al., Eds., pp. 135-142, The Minerals, Metals \& Materials Society, Warrendale, Pa, USA, 2005.

[16] W. Shen, A. B. O. Soboyejo, and W. O. Soboyejo, "Probabilistic modeling of fatigue crack growth in Ti-6Al-4V," International Journal of Fatigue, vol. 23, no. 10, pp. 917-925, 2001.

[17] P. Shi and S. Mahadevan, "Damage tolerance approach for probabilistic pitting corrosion fatigue life prediction," Engineering Fracture Mechanics, vol. 68, no. 13, pp. 1493-1507, 2001.

[18] P. C. McKeighan, A. E. Nicholls, L. C. Perocchi, and R. C. McClung, "Sensing crack nucleation and growth in hard alpha defects embedded in Ti6Al-4V alloy," in Nontraditional Methods of Sensing Stress, Strain, and Damage in Materials and Structures: Second Volume, ASTM STP 1323, G. F. Lucas, P. C. McKeighan, and J. S. Ransom, Eds., American Society for Testing and Materials, West Conshohocken, Pa, USA, 2000.

[19] H. O. Madsen, L. Krenk, and N. C. Lind, Methods of Structural Safety, Dover, New York, NY, USA, 2006.

[20] H. O. Madsen, "Omission sensitivity factors," Structural Safety, vol. 5, no. 1, pp. 35-45, 1988.

[21] I. M. Sobol', "Sensitivity analysis of nonlinear mathematical models," Mathematical Modeling and Computational Experiment, vol. 1, pp. 407-414, 1993, [translation of I. M. Sobol', 
"Sensitivity estimates for nonlinear mathematical models," Matematicheskoe Modelirovanie, vol. 2, pp. 112-118, 1990 (in Russian)].

[22] I. M. Sobol', "Global sensitivity indices for nonlinear mathematical models and their Monte Carlo estimates," Mathematics and Computers in Simulation, vol. 55, no. 1-3, pp. 271-280, 2001.

[23] T. Homma and A. Saltelli, "Importance measures in global sensitivity analysis of nonlinear models," Reliability Engineering and System Safety, vol. 52, no. 1, pp. 1-17, 1996.

[24] W. Chen, R. Jin, and A. Sudjianto, "Analytical variance-based global sensitivity analysis in simulation-based design under uncertainty," Transactions of the ASME: Journal of Mechanical Design, vol. 127, no. 5, pp. 875-886, 2005.

[25] A. K. Karamchandani, New approaches to structural system reliability, Ph.D. thesis, Department of Civil Engineering, Stanford University, Stanford, Calif, USA, 1990.

[26] Y.-T. Wu, "Computational methods for efficient structural reliability and reliability sensitivity analysis," AIAA journal, vol. 32, no. 8, pp. 1717-1723, 1994.

[27] A. H.-S. Ang and W. H. Tang, Probability Concepts in Engineering Planning and Design, Basic Principles, Vol. 1, John Wiley \& Sons, New York, NY, USA, 1975.

[28] H. R. Millwater, M. P. Enright, and S. H. K. Fitch, "A convergent probabilistic technique for risk assessment of gas turbine disks subject to metallurgical defects," in Proceedings of the 43rd AIAA/ASME/ASCE/AHS/ASC Structures, Structural Dynamics, and Materials Conference, Non-Deterministic Approaches Forum, Denver, Colo, USA, April 2002.

[29] L. Huyse and M. P. Enright, "Efficient statistical analysis of failure risk in engine rotor disks using importance sampling techniques," in Proceedings of 44th AIAA/ASME/ASCE/AHS/ASC Structures, Structural Dynamics and Materials Conference, vol. 6, pp. 4040-4050, Norfolk, Va, USA, April 2003. 

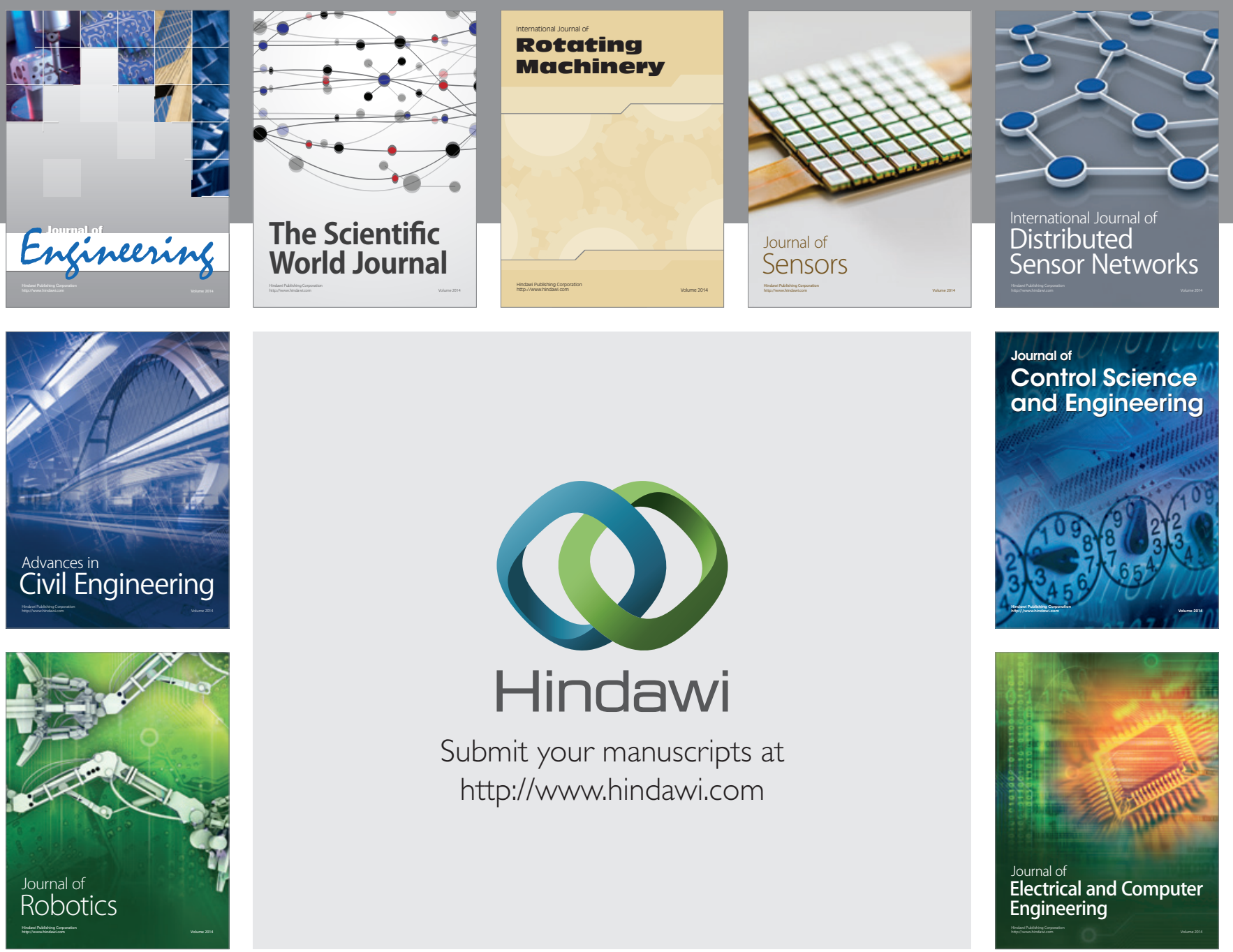

Submit your manuscripts at

http://www.hindawi.com
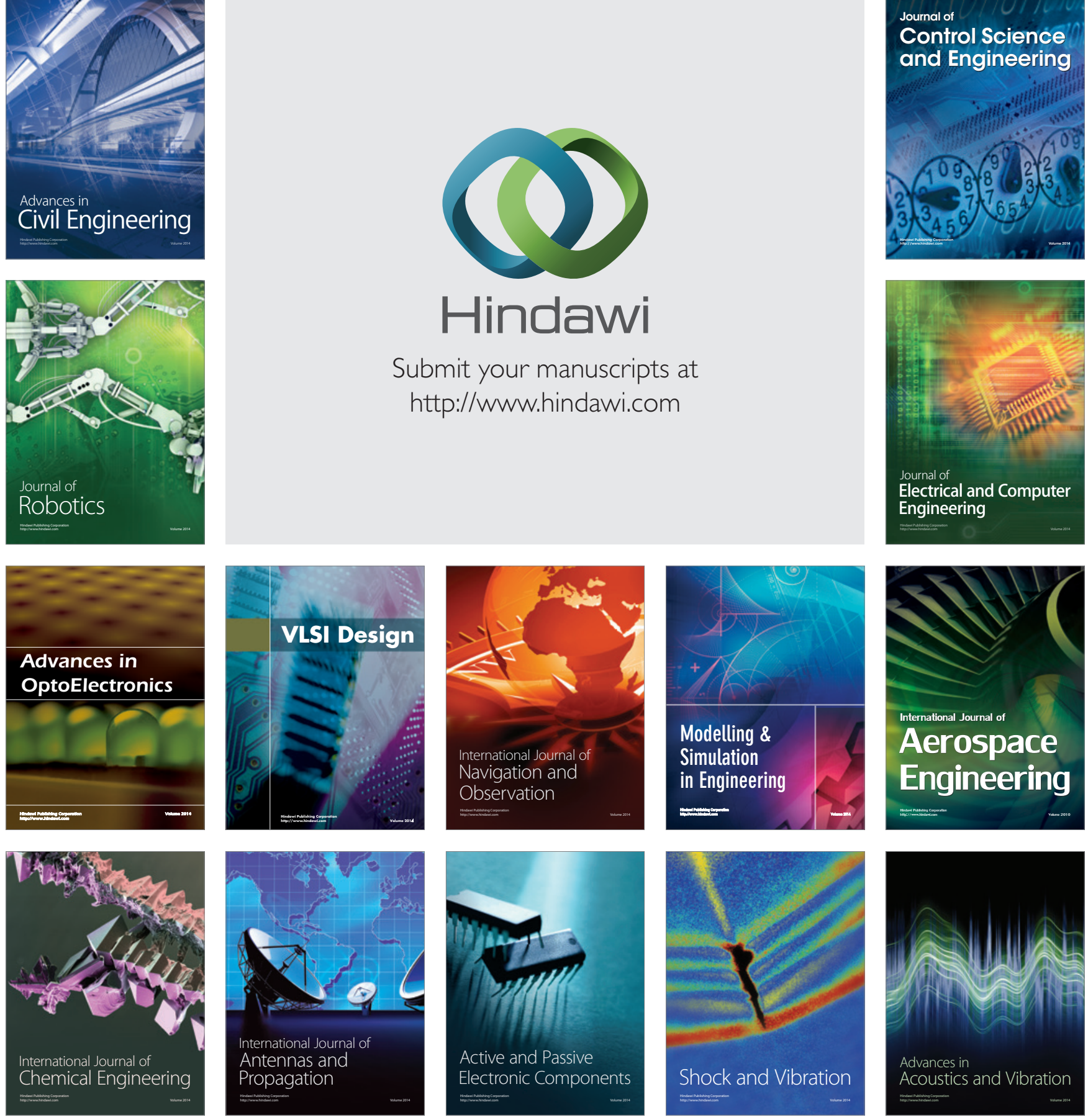